\title{
The MS Choices Survey: findings of a study assessing physician and patient perspectives on living with and managing multiple sclerosis
}

This article was published in the following Dove Press journal:

Patient Preference and Adherence

23 December 201I

Number of times this article has been viewed

\section{Alberto Riñon' \\ Mandy Buch ${ }^{2}$ \\ Derek Holley ${ }^{2}$ \\ Elisabetta Verdun'}

'Merck Serono S.A. - Geneva, Switzerland, a branch of Merck Serono S.A., Coinsins, Switzerland, an affiliate of Merck KGaA,

Darmstadt, Germany;

${ }^{2}$ GfK Healthcare, London, UK

Video abstract

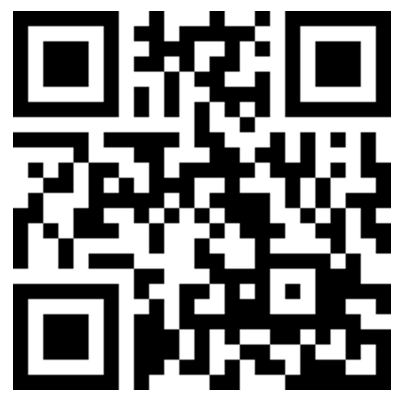

Point your SmartPhone at the code above. If you have a QR code reader the video abstract will appear. Or use: http://dvpr.es/Rinon

Correspondence: Alberto Riñon Merck Serono S.A., 9 Chemin des Mines, 1202 Geneva, Switzerland

Tel +4I 224144206

Fax +4I 224144885

Email alberto.rinon@merckgroup.com
Background: Treatment of multiple sclerosis (MS) with disease-modifying drugs (DMDs) can reduce relapse frequency and delay disability progression. Although adherence to DMDs is difficult to measure accurately, evidence suggests that poor adherence is common and can compromise treatment success. There are likely to be multiple factors underlying poor adherence. To better understand these factors, the global MS Choices Survey investigated patient and physician perspectives regarding key aspects of MS diagnosis, treatment adherence and persistence, and disease management.

Methods: The survey was conducted in seven countries and involved patients with MS (age 18-60 years; MS diagnosis for $\geq 1$ year; current treatment with a DMD) and physicians (neurologist for $3-30$ years; treating $\geq 15$ patients with MS per average month; $>60 \%$ of time spent in clinical practice). Separate questionnaires were used for physicians and patients, each containing approximately 30 questions.

Results: Questionnaires were completed by 331 patients and 280 physicians. Several differences were observed between the responses of patients and physicians, particularly for questions relating to treatment adherence. Overall, the proportion of patients reporting taking a treatment break (31\%) was almost twice that estimated by physicians (on average $17 \%$ ). The reasons cited for poor adherence also differed between patients and physicians. For example, more physicians cited side effects as the main reason for poor patient adherence ( $82 \%)$, than responding patients $(42 \%)$.

Conclusions: Physicians may underestimate the scale of poor adherence to DMDs, which could impact on their assessment of treatment efficacy and result in inappropriate treatment escalation. In addition, disparities were identified between patient and physician responses regarding the underlying reasons for poor adherence. Improvements in the dialog between patients and neurologists may increase adherence to DMDs.

Keywords: multiple sclerosis, treatment initiation, management, patient attitudes, adherence

\section{Introduction}

Multiple sclerosis (MS) is a chronic, heterogeneous, immune-mediated, demyelinating, neurodegenerative disorder of the central nervous system. ${ }^{1}$ It is the most common cause of neurological disability in young adults, ${ }^{2,3}$ affecting an estimated 2.5 million people worldwide. ${ }^{4}$ The most common form of MS follows a relapsing-remitting course, characterized by episodes of neurological dysfunction followed by remission, often with increased levels of residual disability. ${ }^{5,6}$ Although MS is currently incurable, several disease-modifying drugs (DMDs) are available to reduce relapse frequency and severity, and to control disease progression. Most require parenteral administration. 
To gain the full potential benefit from treatment for any illness, including MS, patients need to adhere to their prescribed regimen. The World Health Organization (WHO) defines adherence as “.... the extent to which a person's behaviour taking medication, following a diet, and/or executing lifestyle changes - corresponds with agreed recommendations from a healthcare provider". ${ }^{7}$ The concept of adherence requires the individual to accept the necessity for the medication, and to persist with the therapy. Adherence, therefore, recognizes the patient as the driver of treatment success, ${ }^{8}$ whereas the term "compliance" refers only to the need for patients to follow instructions. Adherence can be described as the sum of acceptance, compliance, and persistence.

Adherence to long-term therapy for chronic diseases tends to be poor; the WHO estimates that the average rate of adherence to medications for chronic diseases is as low as $50 \%$ in developed countries, and may be even lower in developing countries. ${ }^{7}$ These estimates highlight the fact that poor adherence to medication is a serious problem that needs to be addressed.

It is difficult to assess accurately the degree of adherence in patients receiving chronic treatment, and few studies have directly assessed levels of adherence to MS therapy. However, evidence suggests that there is a high level of nonadherence to DMDs in patients with MS. A survey of 798 patients with MS showed that approximately $37 \%$ of patients were nonadherent to DMDs (defined as having missed one or more injections in the previous 4 weeks)..$^{9}$ Another study retrospectively calculated the medication possession ratio (MPR; defined as the number of days' supply of disease-modifying therapy dispensed divided by the number of days evaluated) for 6680 patients with MS who had received at least one DMD between 1999 and 2008. The analysis found that between $52 \%$ and $62 \%$ of patients were adherent to therapy (defined as having an MPR $\geq 80 \%$ ). ${ }^{10} \mathrm{~A}$ similar database analysis of 2446 patients found that $60 \%$ of patients were treatment adherent by the same definition. ${ }^{11}$

Suboptimal levels of treatment adherence may result in suboptimal outcomes. Patients with poor levels of adherence have a higher risk of relapse $\mathrm{e}^{11,12}$ and hospitalization for $\mathrm{MS},{ }^{11}$ as well as incurring higher MS-related medical costs. ${ }^{11}$ Additionally, it is important for patients with MS to maintain high levels of treatment exposure over the long term. Data from the PRISMS (Prevention of Relapses and disability by Interferon beta-1a Subcutaneously in Multiple Sclerosis) long-term follow-up study showed that patients with high levels of exposure to subcutaneous (sc) interferon (IFN) $\beta$-1a (based on cumulative dose or cumulative time on treatment) had lower rates of relapse and Expanded Disability Status Scale progression, and were less likely to convert to secondary progressive MS than were patients with low levels of exposure to sc IFN $\beta-1 \mathrm{a} .{ }^{13}$

In order to improve treatment adherence in patients with a chronic illness, it is vital to first understand why some patients do not take their medication. Several factors appear to interact to drive poor adherence in patients with MS. A 2009 study found that the most common reason for patients missing an injection was simply that they forgot to administer their treatment. ${ }^{9}$ Treatment fatigue was also a factor, with some patients reporting that they did not take their medication because they did not feel like it. ${ }^{9}$ Perceived lack of efficacy is a leading cause of longer interruptions to MS therapy, ${ }^{14}$ and to remain motivated and adhere to therapy, patients need to believe in the value of their treatment and the benefits it confers. ${ }^{8}$ As MS is currently incurable, the DMDs used in MS are intended to slow disease progression, so if patients experience few or no clinical signs of disease, they may not appreciate the importance of remaining adherent to treatment. On the other hand, some patients do experience breakthrough disease while on treatment and consequently may perceive their treatment as ineffective and not worth taking. In these cases it is unclear as to what extent the signs of MS may have worsened without treatment. If patients subsequently become nonadherent, their MS is more likely to be problematic. Adverse events (AEs) associated with treatment can negatively affect adherence to and persistence with MS therapies. ${ }^{9}{ }^{14}$ In the period shortly after treatment initiation, patients are particularly at risk of discontinuation of treatment because of AEs. Later AEs become less of a problem because many of them diminish with time. ${ }^{15}$

Given the difficulty of accurately assessing adherence and the various factors contributing to poor adherence, it is important to better understand the views and experiences of both physicians and patients regarding MS therapies. It is particularly worth noting that patients' perception of their adherence and general experience with therapy may differ from that of their physicians. The MS Choices Survey was commissioned by Merck Serono S.A. - Geneva, Switzerland and performed by GfK Healthcare, London, UK. It investigated patient and physician perspectives on the diagnosis, treatment, and management of MS. Further, the survey sought to identify any discrepancies between the attitudes of patients and those of their physicians. Here, we present the findings of the survey associated with treatment initiation and continuation, as well as factors influencing treatment adherence. 


\section{Methods}

\section{Study design}

\section{Recruitment of respondents}

Patients and physicians from seven countries (Australia, Canada, France, Germany, Italy, Spain, and the UK) were recruited. These countries were selected to give a broad overview of MS from three different continents and to provide a good representation of countries with a high prevalence of MS. ${ }^{16}$ Countries from both northern and southern Europe were represented to enable assessment of different regional attitudes to treatment. Physicians were recruited via an agency online panel; patients were recruited through health care professionals, panels, and other sources such as patient associations.

Inclusion criteria for physicians were that they had been practicing as a neurologist for 3-30 years, treated at least 15 patients with MS in an average month, and spent more than $60 \%$ of their time in clinical practice. Physicians completed a computer-assisted web interview, with enrollment eligibility determined through a series of initial screening questions.

Inclusion criteria for patients were that they were aged 18-60 years, had a diagnosis of MS made at least 1 year previously, and were currently being treated with a DMD. At the time of the survey, all available DMDs were administered parenterally. Patients who completed the survey were not necessarily those treated by the responding physicians.

\section{Data collection}

The survey consisted of two questionnaires: one for physicians and the other for patients (Supplementary Tables 1 and 2). The questionnaires underwent preliminary pilot testing in February 2009 to ensure that respondents fully understood all questions and that the overall flow of the survey was logical and conducive to research aims.

The data were mainly collected between May and June 2009. Data collection complied with international market research regulations for reporting AEs associated with MS therapy, thus ensuring that any previously undisclosed side effects of treatment were acknowledged and that the marketing authorization holders were informed accordingly.

Patients completed a semistructured paper-based questionnaire over approximately 20 minutes. The patient survey comprised 35 closed questions (each with $2-12$ possible responses) and two "free-text response" questions. The physicians' questionnaire was completed online in approximately 15 minutes and comprised 24 closed questions (each with 2-9 possible responses; multiple responses were possible for some questions) and five questions that required physicians to estimate the percentage of their patients involved in each case.
Both questionnaires explored elements of MS diagnosis, treatment, and disease management to obtain greater detail on key areas, such as the current needs and future developments of treatment for MS. Research was coordinated by an international health care market research agency, which collated responses from the survey into a wider research database, and conducted detailed analysis to generate qualitative and quantitative outputs.

\section{Results \\ Demographics}

In total, 280 physicians completed the survey (Australia, $\mathrm{n}=10$; Canada, $\mathrm{n}=20$; France, $\mathrm{n}=50$; Germany, $\mathrm{n}=50$; Italy, $\mathrm{n}=50$; Spain, $\mathrm{n}=50$; UK, $\mathrm{n}=50$ ). Questionnaires were completed by 331 patients (Australia, $\mathrm{n}=30$; Canada, $\mathrm{n}=51$; France, $\mathrm{n}=50$; Germany, $\mathrm{n}=50$; Italy, $\mathrm{n}=50$; Spain, $\mathrm{n}=50 ; \mathrm{UK}, \mathrm{n}=50$ ).

\section{Patient involvement in treatment decisions}

In total, 58\% (161/280) of physicians believed that patients should select their treatment in partnership with their medical team; however, when asked "How involved are your MS patients in the treatment decision-making process?", only $47 \%(131 / 280)$ of these physicians stated that their patients were fully involved. This proportion varied among countries, with Canada $(75 \% ; 15 / 20)$ appearing to have the highest level of patient involvement in the decisionmaking process, followed by the UK $(62 \% ; 31 / 50)$. This proportion was lowest in Spain, where only 20\% (10/50) of physicians stated that their patients were fully involved in the process.

Overall, 23\% (77/331) of patients felt that they had discussed treatment options with their medical team, and had themselves been responsible for the selection of their treatment. Canada $(53 \% ; 27 / 51)$ had the highest proportion of patients selecting their own treatment, followed by the UK $(30 \% ; 15 / 50)$, Germany $(24 \% ; 12 / 50)$, France $(20 \% ; 10 / 50)$, Australia $(13 \% ; 4 / 30)$, Spain $(12 \% ; 6 / 50)$, and Italy $(6 \% ; 3 / 50)$. Twenty-eight percent $(93 / 331)$ of patients reported that their physician or nurse had selected their treatment without any patient discussion. Regardless of their involvement in the selection of their therapy, most patients $(89 \%$; 295/331) reported being aware of both the benefits and possible side effects of their current MS treatment, and being aware of the existence of other MS therapies $(84 \% ; 278 / 331)$. 
Table I Initiation of disease-modifying drugs (DMDs): patient and physician responses, by country

Physician question:

On average, how long following the diagnosis of MS do you usually start to actively treat a patient with pharmacotherapies/DMDs?

Patient question:

How long following your diagnosis did you start undergoing treatment for your MS?

\begin{tabular}{|c|c|c|c|c|c|c|c|c|}
\hline & \multicolumn{2}{|c|}{ Within 2 months } & \multicolumn{2}{|c|}{ Within 3-6 months } & \multicolumn{2}{|c|}{ Within 7-I 2 months } & \multicolumn{2}{|c|}{ Greater than 12 months } \\
\hline & Physician (\%) & Patient (\%) & Physician (\%) & Patient (\%) & Physician (\%) & Patient (\%) & Physician (\%) & Patient (\%) \\
\hline Australia & 80 & 37 & 10 & 10 & 0 & 13 & 10 & 40 \\
\hline Canada & 45 & 31 & 45 & 22 & 10 & 12 & 0 & 35 \\
\hline France & 48 & 42 & 44 & 20 & 2 & 12 & 6 & 26 \\
\hline Germany & 64 & 24 & 32 & 26 & 4 & 10 & 0 & 40 \\
\hline Italy & 56 & 42 & 34 & 30 & 8 & 12 & 2 & 16 \\
\hline Spain ${ }^{a}$ & 38 & 22 & 44 & 16 & 16 & 10 & 2 & 50 \\
\hline UK & 24 & 14 & 44 & 20 & 24 & 18 & 8 & 48 \\
\hline Overall & 47 & 30 & 39 & 21 & 10 & 12 & 4 & 36 \\
\hline
\end{tabular}

Notes: ${ }^{2} \%$ of patients responded 'Don't know'; $n=33$ I for total patients; $n=280$ for total physicians.

\section{Timing of treatment initiation}

There were considerable differences between responses from physicians and patients to questions regarding the timing of treatment initiation. The findings from the physician and patient questionnaires regarding time between diagnosis and treatment initiation, by country, are presented in Table 1.

Across all seven countries, 86\% (241/280) of physicians responded that, on average, treatment was initiated (pharmacotherapy/DMDs) within 6 months of diagnosis; 4\% $(10 / 280)$ stated that they generally initiated treatment more than 12 months after a diagnosis of MS. Physician response to this question varied across countries, with $96 \%$ (48/50) of physicians from Germany reporting that they initiate therapy within 6 months of diagnosis, compared with $68 \%(34 / 50)$ of those from the UK. In contrast, only 51\% (169/331) of all patients reported starting treatment within 6 months of their diagnosis. The corresponding proportion from Germany was $50 \%(25 / 50)$ and from the UK 34\% (17/50). Furthermore, $36 \%(120 / 331)$ of patients said that their treatment was initiated more than 12 months after diagnosis. Patient responses to this question also varied across countries: Spain had the highest proportion of patients $(50 \% ; 25 / 50)$ reporting initiating

Table 2 Summary of treatment interruptions and discontinuations: patient and physician responses, by country Physician questions:

I. Approximately what percentage, if any, of all your treated MS patients take a treatment break?

2. Approximately what percentage, if any, of all your treated MS patients stop their treatment?

Patient questions:

I. Have you ever taken a break from your MS treatment, ie, where you have actively decided not to take your treatment as opposed to forgetting to take it and which could last I day or longer?

2. Have you ever stopped taking your MS treatment?

\begin{tabular}{lllll}
\hline & \multicolumn{2}{l}{$\begin{array}{l}\text { Patients taking a break from treatment } \\
\text { (mean scores) }\end{array}$} & \multicolumn{2}{l}{$\begin{array}{l}\text { Patients discontinuing treatment } \\
\text { (mean scores) }\end{array}$} \\
\cline { 2 - 4 } & Physician (\%) & Patient (\%) & Physician (\%) & Patient (\%) \\
\hline Australia & 17 & 47 & 12 & 30 \\
Canada & 17 & 37 & 14 & 27 \\
France & 15 & 44 & 10 & 15 \\
Germany & 18 & 16 & 13 & 10 \\
Italy & 20 & 40 & 12 & 12 \\
Spain & 14 & 18 & 17 & 12 \\
UK & 18 & 20 & 14 & 19 \\
Overall & 17 & 31 & & \\
\hline N
\end{tabular}

Notes: $n=33$ I for total patients; $n=280$ for total physicians. 


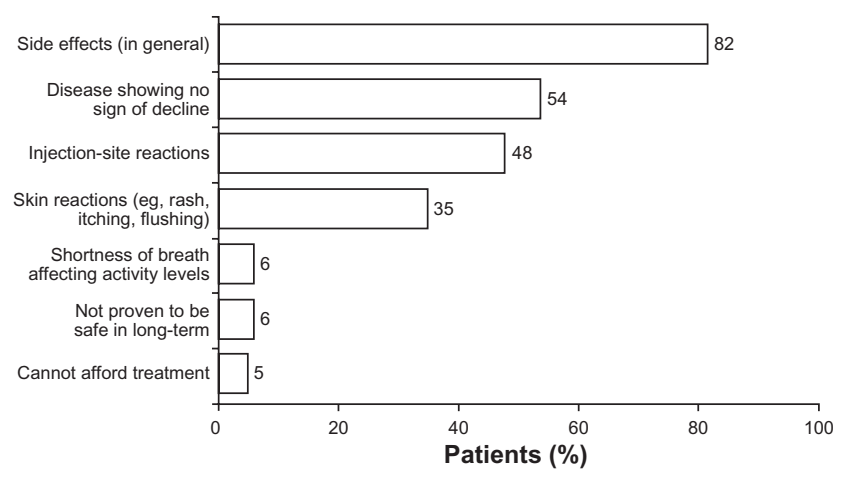

Figure I Main reasons why patients may take a break or stop their MS treatment, as rated by physicians.

Notes: $\mathrm{n}=277$ (total number of physicians with patients who had taken a break from, or stopped, their MS therapy). 'Other' responses not shown because the number of responses was too small.

therapy more than 12 months after diagnosis, compared with $16 \%(8 / 50)$ from Italy.

\section{Treatment interruptions and discontinuations}

Table 2 provides a summary of patient and physician responses, by country, of treatment breaks and discontinuations.

On average, physicians estimated that approximately $17 \%$ of all their treated patients with MS take a break from treatment, and that $14 \%$ discontinue treatment altogether. In contrast, $31 \%$ of responding patients reported having taken a deliberate break from treatment of 1 day or longer, and $19 \%$ reported that they had stopped taking their MS treatment completely. The proportion of patients having taken a

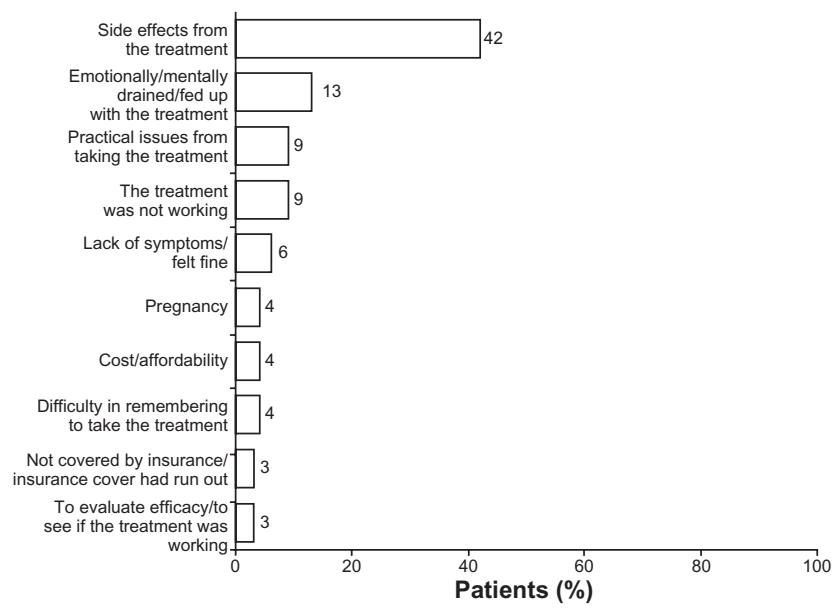

Figure 2 Main reasons for taking a break or stopping their MS treatment, as rated by patients.

Notes: $\mathrm{n}=113$ (total number of patients who had ever taken a break from or stopped their MS treatment). break from treatment was highest in Australia (47\%; 14/30) and lowest in Germany $(16 \% ; 8 / 50)$. Almost all physicians in this survey reported having at least one patient who had taken a break from and/or stopped their MS therapy (93\%; $260 / 280$ and $98 \% ; 273 / 280$, respectively). More than half of physicians $(59 \% ; 166 / 280)$ responded "No" to the question "In general, do you find compliance an issue when treating MS patients?"

Physicians who reported having patients taking a break from, or discontinuing, treatment $(n=277)$ ranked "Side effects (in general)" as the main reason that patients may take a break or stop their MS treatment ( $82 \%$ of respondents), followed by "Disease showing no sign of decline" (54\% of respondents; see Figure 1).

Only $42 \%$ of patients who reported taking a treatment break or stopping their treatment $(n=113)$ cited "Side effects from the treatment" as the main reason for this change, followed by being "Emotionally/mentally drained/ fed up with the treatment", "Practical issues from taking the treatment", and "The treatment was not working" (see Figure 2).

Overall, just over half $(56 \% ; 187 / 331)$ of all patients in this survey reported discussing aspects of their MS treatment other than side effects with their physician or nurse, although this varied considerably across the different countries involved. For example, 84\% (42/50) of UK patients reported taking this action compared with $37 \%$ (11/30) of Australian respondents.

\section{Discussion}

This study was designed to assess physicians' and patients' perceptions of the management of MS and their attitudes towards treatment. It is generally accepted that good patient-physician communication can help to maintain a patient's motivation for, and adherence to, DMD therapy. ${ }^{17}$ The findings reported in this study suggest that there is often substantial disparity between the views of physicians and those of patients with MS, underlining the need for open communication and cooperation between the patient and the health care team.

There is evidence that the benefits of DMDs are greatest when treatment is started early in the disease course. ${ }^{18}$ In the majority of countries participating in this survey, treatment with DMDs was initiated within 12 months of MS diagnosis. This finding was not, however, consistent in all instances, and there were differences among countries. This may be due to cultural differences and variations between the health care systems of the countries involved in the survey. 
Additionally, some physicians advocate a gap of some months between diagnosis and treatment initiation, to allow patients to assimilate the implications of their diagnosis and to accept the need for treatment. Interestingly, there was considerable disparity between the physician and patient responses regarding the proportion of patients waiting over 12 months to initiate treatment, the potential reasons for which are currently unknown.

Although the importance of involving the patient in therapeutic decision making is widely recognized, one in four patients in this study responded that their treatment options had not been discussed with them prior to the selection of a DMD by their medical team. In some cases this may have reflected the preference of patients to allow their physicians to make treatment decisions. Additionally, while it is important for patients to be engaged in the decision-making process, their disease characteristics may, to some extent, dictate which therapy is appropriate.

Our results show that, although nearly all physicians recognized that at least one of their patients had taken a break from medication, the true scale of nonadherence was generally underestimated by physicians, although there was considerable variability between countries in the disparity between physician and patient responses regarding treatment persistence. While the degree to which patients adhere to their medication regimen is difficult to assess and quantify accurately, this study found that gaps in treatment and discontinuation were common, a result that is in line with previous data. ${ }^{14,15,19,20}$ In this study, the majority of physicians did not find compliance a problem; they felt their patients were able to follow instructions. This, however, would seem to be at odds with the finding of a high proportion of patients taking treatment breaks.

As previously observed, ${ }^{15}$ AEs were recognized by both patients and physicians as having a negative impact on adherence. There is a need for education programs aimed at increasing both physician and patient awareness of methods for managing AEs associated with DMDs.

Although our study contributes to the understanding of the issues facing patients and their physicians regarding the treatment of MS, certain limitations must be acknowledged. It is difficult to accurately compare the responses of the physicians with those of the patients: the patient population was a general sample from each country, rather than from the same centers as the physicians. This may have affected the finding that patient and physician perceptions differed. Furthermore, the inclusion criteria selected for centers treating relatively high proportions of patients with MS. It is possible that any disconnect between the attitudes of physicians and those of patients may be underestimated as a result - physicians treating fewer patients with MS may be less aware of adherence issues than those who more regularly treat MS. In some cases, the questions asked of the patients were necessarily different from those asked of the physicians, and the two questionnaires were administered in two different formats (one written, one completed online). Additionally, data are not available to determine any disease characteristics that may have led to differences in response. Respondents' interpretation of some of the terms used in the questionnaires (such as "compliance", "treatment break", and "adherence") may have varied as the terms were not defined in the questionnaires. Furthermore, owing to this being an international survey, the observed differences in responses for some questions may have been a result of regional and national variations in health care provision, such as the availability of certain medications.

At the time of this survey, all available DMDs were administered parenterally. It remains to be seen whether the recent advent of oral therapies for MS will affect rates of treatment adherence, but experience in other therapy areas shows that, as with injectable drugs, adherence to oral therapies tends to be suboptimal. ${ }^{21}$

In summary, these findings not only support previous suggestions that patient adherence to MS therapy is suboptimal, but also highlight that physicians may underestimate and undervalue the actual levels of adherence among their patients. This underestimation may prevent physicians from accurately assessing the efficacy of the treatment prescribed, which, in turn, could lead to inappropriate treatment escalation in patients who appear to be responding poorly to first-line therapy. Improving the dialogue between patients and health care professionals may support greater adherence to DMD therapy and, ultimately, improve outcomes.

\section{Acknowledgments}

The authors thank Steve Smith and Anna Palagyi of Caudex Medical Ltd, Oxford, UK (supported by Merck Serono S.A. - Geneva, Switzerland, a branch of Merck Serono S.A., Coinsins, Switzerland, an affiliate of Merck KGaA, Darmstadt, Germany), for assistance with the preparation of the initial draft of the manuscript and for collating input from all authors (SS), and for editing for English and assistance with preparation for submission (AP). 


\section{Disclosure}

This study was commissioned and supported by Merck Serono S.A. - Geneva, Switzerland, a branch of Merck Serono S.A., Coinsins, Switzerland, an affiliate of Merck KGaA, Darmstadt, Germany, with research conducted by GfK Healthcare, London, UK. AR and EV are employees of Merck Serono S.A. - Geneva, Switzerland, a branch of Merck Serono S.A., Coinsins, Switzerland, an affiliate of Merck KGaA, Darmstadt, Germany. MB and DH are employees of GfK Healthcare, London, UK.

\section{References}

1. Bennett JL, Stuve O. Update on inflammation, neurodegeneration, and immunoregulation in multiple sclerosis: therapeutic implications. Clin Neuropharmacol. 2009;32:121-132.

2. McDonald WI. Relapse, remission, and progression in multiple sclerosis. N Engl J Med. 2000;343:1486-1487.

3. Murray TJ. Diagnosis and treatment of multiple sclerosis. BMJ. 2006;332:525-527.

4. Compston A, Confavreux C, Lassmann H. McAlpine's Multiple Sclerosis. 4th ed. Philadelphia, PA: Churchill Livingstone; 2006.

5. Confavreux C, Vukusic S, Moreau T, Adeleine P. Relapses and progression of disability in multiple sclerosis. $N$ Engl J Med. 2000;343: 1430-1438.

6. Weinshenker BG, Bass B, Rice GP, et al. The natural history of multiple sclerosis: a geographically based study. 1. Clinical course and disability. Brain. 1989;112(Pt 1):133-146.

7. World Health Organization. Adherence to long-term therapies: evidence for action. Available from: http://www.who.int/chp/knowledge/publications/ adherence_report/en/index.html. Accessed August 21, 2011.

8. Lugaresi A. Addressing the need for increased adherence to multiple sclerosis therapy: can delivery technology enhance patient motivation? Expert Opin Drug Deliv. 2009;6:995-1002.

9. Treadaway K, Cutter G, Salter A, et al. Factors that influence adherence with disease-modifying therapy in MS. J Neurol. 2009;256:568-576.
10. Halpern R, Agarwal S, Dembek C, Borton L, Lopez-Bresnahan M Comparison of adherence and persistence among multiple sclerosis patients treated with disease-modifying therapies: a retrospective administrative claims analysis. Patient Prefer Adherence. 2011;5:73-84.

11. Tan H, Cai Q, Agarwal S, Stephenson JJ, Kamat S. Impact of adherence to disease-modifying therapies on clinical and economic outcomes among patients with multiple sclerosis. Adv Ther. 2011;28:51-61.

12. Al-Sabbagh A, Bennet R, Kozma C, Meletiche D. Medication gaps in disease-modifying therapy for multiple sclerosis are associated with an increased risk of relapse: findings from a national managed care database. J Neurol. 2008;255(Suppl 2):S79.

13. Uitdehaag B, Constantinescu C, Cornelisse $\mathrm{P}$, et al. Impact of exposure to interferon beta-1a on outcomes in patients with relapsing-remitting multiple sclerosis: exploratory analyses from the PRISMS long-term follow-up study. Ther Adv Neurol Disord. 2011;4:3-14.

14. Tremlett HL, Oger J. Interrupted therapy: stopping and switching of the beta-interferons prescribed for MS. Neurology. 2003;61:551-554.

15. O'Rourke KE, Hutchinson M. Stopping beta-interferon therapy in multiple sclerosis: an analysis of stopping patterns. Mult Scler. 2005;11:46-50.

16. Multiple Sclerosis International Federation. Atlas of MS Database 2007 Available from: http://www.atlasofms.org/query.aspx?pq=yes\&s=1\&q= 3\&r=Global\&year=2007. Accessed August 21, 2011.

17. Tremlett H, Vander Mei I, Pittas F, et al. Adherence to the immunomodulatory drugs for multiple sclerosis: contrasting factors affect stopping drug and missing doses. Pharmacoepidemiol Drug Saf. 2008; 17:565-576.

18. Multiple Sclerosis International Federation. MS: the Guide to Treatment and Management 2008. Available from: http://www.msif.org/ en/resources/msif_resources/msif_publications/ms_the_guide_to_ treatment_and_management/treatments_affecting_longterm_course_ of_disease/index.html. Accessed August 21, 2011.

19. Portaccio E, Zipoli V, Siracusa G, Sorbi S, Amato MP. Long-term adherence to interferon beta therapy in relapsing-remitting multiple sclerosis. Eur Neurol. 2008;59:131-135.

20. Ri J, Porcel J, Tellez N, et al. Factors related with treatment adherence to interferon beta and glatiramer acetate therapy in multiple sclerosis. Mult Scler. 2005;11:306-309.

21. Osterberg L, Blaschke T. Adherence to medication. N Engl J Med. 2005; 353:487-497. 
Table SI Questions included in physicians' questionnaire

\section{Physician questions}

I. On average, how long following the diagnosis of MS do you usually start to actively treat a patient with pharmacotherapies/disease-modifying drugs?

a. Within 2 months

b. Within 3-6 months

c. Within 7-12 months

d. Greater than 12 months following diagnosis

2. In general, to what extent do you think patients should be involved in choosing their MS treatment?

a. Not at all, physician only should make that decision

b. Treatment options discussed, but ultimately the physician should decide on the best option for the patient

c. Patients should select their treatment in partnership with their medical team

3. In general, how involved are your MS patients in the treatment decision-making process?

a. Fully involved

b. Fairly involved

c. Not involved at all

4. In your opinion, what are the three most important factors to consider when prescribing an MS treatment?

a. Effectiveness (based on clinical trials)

b. Side-effect profile

c. Long-term safety profile

d. Cost

e. Other, please specify

5. In your opinion, which three factors do patients consider most important when deciding on a treatment?

a. Overall efficacy

b. Side-effect profile

c. Method of administration

d. Frequency of treatment

e. Long-term safety profile

f. Other, please specify

6. How well do your patients understand the relative benefits and associated risks of currently available MS treatments?

a. Very well

b. Fairly well

c. Not very well

d. Not at all well

7. Please indicate what side effects of treatment mainly affect the patients' adherence to their MS therapy?
a. Injection-site reactions
b. Flu-like symptoms
c. Skin-site reactions
d. Mood changes
e. Lipoatrophy
f. Chest tightness
g. Increased muscle spasms
h. Shortness of breath
i. Other, please specify

8. Which side effect of treatment has the greatest impact on adherence to MS therapy?
a. Injection-site reactions
b. Flu-like symptoms
c. Skin-site reactions
d. Mood changes
e. Lipoatrophy
f. Chest tightness
g. Increased muscle spasms
h. Shortness of breath
i. Other

9. For those selecting (a) as a response to question 7 or 8: Approximately what percentage, if any, of all your MS patients complain of injection-site reactions? 
Table SI (Continued)

\section{Physician questions}

10. In general, do you find compliance an issue when treating MS patients?

a. Yes

b. No

II. Approximately what percentage, if any, of all your treated MS patients take a treatment break?

12. Approximately what percentage, if any, of all your treated MS patients stop their treatment?

13. For those responding with $>0 \%$ to question II or 12: What are the main reasons for patients to take a break or stop their MS treatment? a. Side effects (in general)

b. Disease showing no sign of decline

c. Injection-site reactions

d. Skin reactions (for example, rash, itching, flushing)

e. Shortness of breath affecting activity levels

f. Not proven to be safe in long-term

g. Cannot afford treatment

h. Other, please specify

14. For those responding with $>0 \%$ to question II or I2: Do physical or psychological factors (ie, patient's belief in his/her MS treatment) predominantly affect the patient's decision to take a break or stop his/her treatment?

a. Physical and psychological factors in equal measures

b. Psychological factors

c. Physical factors

15. What (other) aspects, if any, do your MS patients generally find challenging about their current treatment regimes?

a. Side effects (in general)

b. Injection-related issues

c. Slow response to treatment

d. Maintaining a medication schedule

e. Affording medication

f. Other, please specify

16. What do you think MS patients would cite as the most important factor that would improve their compliance in taking the treatment?

a. No more injections

b. Taking treatment less frequently

c. If their medication improves overall well-being

d. Easier to take

e. Other, please specify

17. If a patient could take the treatment less frequently, how much might the compliance to the treatment change?

a. Improve significantly

b. Improve moderately

c. No change/neither deteriorate nor improve

d. Deteriorate moderately

e. Deteriorate significantly

18. Approximately what percentage, if any, of all your treated MS patients have switched treatments (following initial prescription)?

19. For those responding $>0 \%$ to question 18: What are the main reasons for patients switching to a new treatment? a. Side effects (in general)

b. Improved efficacy

c. Improved injection-site reactions

d. Less frequent treatment

e. Greater long-term safety profile

f. Maintaining a medication schedule

g. Other, please specify

20. Apart from efficacy, do you regularly review your MS patients' treatment?

a. Yes

b. No 


\section{Table SI (Continued)}

\section{Physician questions}

21. For those responding (a) to question 20: On average, how often do you review the treatment for your

MS patients?
a. More often than every 6 months
b. Between 6 months but less than I year
c. Between I year but less than 2 years
d. Between 2 years but less than 3 years
e. Between 3 years but less than 4 years
f. Between 4 years but less than 5 years
g. Every 5 or more years
h. Don't know/varies

22. Approximately what percentage, if any, of all your MS patients ever initiate a discussion about possible other treatments for their condition?

23. Would you change your first-line MS treatment for another therapy that a patient may find easier to comply with (yes, would change/no, would not change), if it had ...

a. ... Greater efficacy

b. ... Fewer side effects

c. ... Greater long-term safety profile

d. ... Different method of administration

e. ... More side effects

f. ... Reduced efficacy

24. What specific information or services, if any, do you offer your MS patients on how they may maintain or improve their overall quality of life?

a. Moral support/advice

b. Refer to local MS support services

c. Support/information from pharmaceutical company

d. Refer to national/local patient group

e. Other, please specify

25. Specifically, what information or services, if any, do you offer your MS patients on helping them cope with the physical disabilities caused by their disease?

a. Refer to a physiotherapist

b. Refer to the multidisciplinary team

c. Refer to appropriate healthcare professionals for further assistance

d. Other, please specify

26. And specifically, what information or services, if any, do you offer your MS patients on helping them cope with the mental impairments caused by their disease?

a. Refer to a counselor/counseling service

b. Refer to MS support services

c. Refer to multidisciplinary team

d. Other, please specify

27. In general, do you think pharmaceutical companies give sufficient consideration with regards to the needs of MS patients when developing new treatment options?

a. Yes

b. No

28. Which treatment/management innovations do you believe would be likely to benefit MS patients the most if they were available?
a. Oral therapy
b. New specific biological therapies
c. Mobility/disability aids
d. Stem cell transplant
e. Other, please specify

29. For those responding (a) to question 28: Why do you say that an oral therapy would be most likely to benefit MS patients if it was available? (Free text response) 
Table S2 Questions included in patients' questionnaire

\section{Patient questions}

I. How long following your diagnosis did you start undergoing treatment for your MS?

a. Within 2 months

b. Within 3-6 months

c. Within 7-12 months

d. Greater than 12 months

e. Don't know

2. How involved were you in choosing your MS treatment?

a. Your doctor/nurse selected your treatment (without discussing any treatment options with you)

b. Your doctor/nurse and you discussed different treatment options and he/she recommended the one to take

c. Your doctor/nurse and you discussed different treatment options and you decided which one to take

3. For those responding (b) or (c) to question 2: When you were discussing with your doctor/nurse which MS treatment to take, what were the most important factors for you to consider?

a. How effective the treatment would be

b. Possible side effects from taking the treatment

c. How the treatment would be taken

d. How it could affect your quality of life

e. Long-term safety profile of the treatment

f. Other, please specify

g. Don't know

4. For those responding (b) or (c) to question 2: Please rate how important each of the following factors were to you when deciding which MS treatment to take? (Scale from I = 'not at all important' to 5 = 'extremely important')

a. How effective the treatment would be

b. Possible side effects from taking the treatment

c. How it could affect your quality of life

d. How the treatment would be taken

e. Long-term safety profile of the treatment

f. Other, from Q3

5. Are you aware of both the benefits and possible side effects of your current MS treatment?

a. Yes, you know both the benefits and side effects

b. No, you only know the benefits

c. No, you only know the side effects

d. No, you do not know about either

e. Don't know

6. For those responding (a) or (b) to question 5: What do you consider are the three main benefits from taking your current MS treatment?

a. Longer between/less frequency of attacks/episodes/flare-ups

b. Reduction of progression of the disease

c. Maintains current status/condition

d. Decrease in severity of attacks/episodes/flare-ups

e. Prevents symptoms getting worse

f. Long-term disability is reduced/prolongs time to long-term disability

g. Long-term safety profile

h. Other, please specify

i. Don't know

7. Apart from what you are currently taking, are you aware of any other available MS treatment(s)?

a. Yes

b. No

c. Don't know

8. For those responding (a) to question 7: How much do you know about the benefits and possible side effects of other MS treatment(s)?

a. You know a lot about them

b. You know something about them

c. You know little about them

d. You know nothing about them

e. Don't know

9. Have you ever experienced any side effects from your MS treatment?

a. No

b. Yes 
Table S2 (Continued)

\section{Patient questions}

10. For those responding (b) to question 9: Have these side effects from your MS treatment affected your daily life?
a. No
b. Yes

II. For those responding (b) to question 10: Which side effects from your MS treatment have affected your daily life the most?

a. Flu-like symptoms

b. Tiredness/lethargy

c. Injection-site reactions (eg, redness, soreness, scarring)

d. Mood changes

e. Lack of sleep/difficulty sleeping

f. Skin reactions (itching, rash, flushing of skin)

g. Increased muscle spasms

h. Chest tightness

i. Shortness of breath

j. Other, please specify

k. Don't know

12. For those responding (c) to question II: To what extent do injection site reactions affect your daily life? (Scale from I = 'slightly affected' to $5=$ 'greatly affected')

13. Have you ever taken a break from your MS treatment, ie, where you have actively decided not to take your treatment as opposed to forgetting to take it and which could last I day or longer?

a. Yes

b. No

14. Have you ever stopped taking your MS treatment?

a. Yes

15. For those responding (a) to question 13 or 14: What led to your decision to take a break or stop your MS treatment?

a. Physical factors

b. Psychological factors

c. Physical and psychological factors in equal measures

d. Don't know

16. For those responding (a) to question 13 or 14: Specifically, what were the main reasons for taking a break or stopping your MS treatment?

a. Side effects from the treatment

b. The treatment was not working

c. Practical issues from taking the treatment

d. Difficulty in remembering to take the treatment

e. Convenience

f. Treatment was not proven to be safe over long-term use

g. Other, please specify

h. Don't know

17. For those responding (a) to question 16: Specifically, what side effects did you experience which made you take a break or stop your MS treatment?

a. Tiredness/lethargy

b. Injection-site reactions (eg, redness, soreness, scarring)

c. Flu-like symptoms

d. Skin reactions (itching, rash, flushing of skin)

e. Mood changes

f. Shortness of breath

g. Increased muscle spasms

h. Chest tightness

i. Lack of sleep/difficulty sleeping

j. Other, please specify

k. Don't know 
Table S2 (Continued)

\section{Patient questions}

18. What do you consider is the most difficult aspect of taking your current MS treatment?

a. Fitting the injection around your lifestyle (work, holiday, leisure, etc)

b. Doing your daily activities while trying to manage the side effects from taking the treatment

c. Being physically able to inject it

d. Being emotionally able to inject it

e. Managing the emotional side effects from taking the treatment

f. Practically managing the injections (storage, disposing of needles, etc)

g. None in particular

h. Don't know

19. Apart from any side effects of the MS treatment that you may have experienced, do you ever discuss any other aspect of your MS treatment with your doctor/nurse?
a. Yes
b. No
c. Don't know

20. What do you consider are the most important factors concerning your MS treatment that can help improve your quality of life?

a. No more injections/take alternative form of treatment

b. Good safety profile when used over a long period of time

c. None or fewer side effects

d. Less frequent dosing

21. Please rank these factors in order of their importance with regards to their affect on improving your likelihood to take your MS treatment (rank I to 4: I being 'most important', 4 being 'least important')

a. No more injections/take alternative form of treatment

b. Good safety profile when used over a long period of time

c. None or fewer side effects

d. Less frequent dosing

22. How much would your life in general change if you could take your treatment less frequently?
a. Improve significantly
b. Improve moderately
c. No change at all
d. Worsen
e. Don't know

23. How much would your emotional well-being change if you could take your treatment less frequently?
a. Improve significantly
b. Improve moderately
c. No change at all
d. Worsen
e. Don't know

24. What sources or services, if any, do you use to get more information on how to manage your MS treatment more effectively?

a. Neurologist

b. MS Society/associations

c. Additional online source/website

d. Literature (books, magazines, etc)

e. General practitioner (GP)

f. MS nurse

g. Family/friends

h. Literature from pharmaceutical/drug company/manufacturer

i. Pharmaceutical/drug company/manufacturer's website

j. Other, please specify

k. None

I. Don't know

25. Does your doctor/nurse regularly review your MS treatment?
a. Yes
b. No
c. Don't know 


\section{Table S2 (Continued)}

\section{Patient questions}

26. For those responding (a) to question 25: On average, how often does your doctor/nurse review your

MS treatment with you?

a. More often than every 3 months

b. Every 3-6 months

c. Every 7-9 months

d. Every 10-12 months (once a year)

e. Every 13-23 months

f. Once every 24 months ( 2 years)

g. Less often than once every 2 years

h. Don't know

27. Have you ever initiated a discussion with your doctor/nurse about other MS treatments?
a. Yes
b. No
c. Don't know

28. Have you ever asked your doctor/nurse to change your MS treatment?
a. Yes
b. No
c. Don't know

29. For those responding (a) to question 28: What was your doctor's/nurse's response to your request to change your MS treatment? What did he/she say and do? (Free text response)

30. In general, to what extent, if at all, has your physical well-being been affected by your MS?
a. Improved
b. Not changed
c. Worsened moderately
d. Worsened significantly
e. Don't know

3I. In general, to what extent, if at all, has your emotional well-being been affected by your MS?
a. Improved
b. Not changed
c. Worsened moderately
d. Worsened significantly
e. Don't know

32. In general, how much has your MS affected your overall quality of life?
a. Improved
b. Not changed
c. Worsened moderately
d. Worsened significantly
e. Don't know

33. Which new treatment/innovation do you believe would be likely to benefit you the most, as an MS patient,

if it was available?
a. Oral therapy (tablet/capsule/pill, etc)
b. Stem cell therapy/transplant
a. Monthly or annual intravenous injections/infusion
b. New monoclonal antibodies
c. Other
d. Don't know

34. Do you think pharmaceutical/drug companies give sufficient consideration to the needs of MS patients when developing new treatments?
a. Yes
b. No
c. Don't know

35. For those responding (b) to question 33: Why do you say that? (Free text response) 
Table S2 (Continued)

\section{Patient questions}

36. For those responding (a) to question 33: Why would an oral therapy benefit you the most?
a. No more injections
a. Take treatment less frequently
b. Easier to take/remember to take
c. Other
d. Don't know

37. If an oral treatment was available but less effective than the current injectable forms, if you had the choice, would you prefer to take either the oral or injectable form?
a. Injectable form
b. Oral form
c. No preference/don't mind either
d. Don't know

Patient Preference and Adherence

\section{Publish your work in this journal}

Patient Preference and Adherence is an international, peer-reviewed, open access journal focusing on the growing importance of patient preference and adherence throughout the therapeutic continuum. Patient satisfaction, acceptability, quality of life, compliance, persistence and their role in developing new therapeutic modalities and compounds to optimize clinical outcomes for existing disease states are major areas of interest. This journal has been accepted for indexing on PubMed Central. The manuscript management system is completely online and includes a very quick and fair peer-review system. Visit http://www.dovepress.com/ testimonials.php to read real quotes from published authors.

\footnotetext{
Submit your manuscript here: http://www.dovepress.com/patient-preference-and-adherence-journal
} 